\title{
Electronic Materials Conference Held in Newark
}

The second International Conference on Electronic Materials (ICEM-90) was held in Newark, New Jersey, September 17-19, 1990, under the co-sponsorship of the Materials Research Society, the European Materials Research Society, and the Japan Society of Applied Physics.

The conference focused on emerging electronic materials impacting the electronics industry in the 21st century. Four topical areas were covered: materials for high $T_{c}$ superconducting electronics, materials for optoelectronics, advanced thin film technology, and diamond for electronic and optical applications. To maximize cross-disciplinary interactions no parallel sessions were held, and poster sessions were interspersed between oral sessions. $\mathrm{H}$. Watanabe of NEC Corporation gave the after-banquet talk, "Toward a SingleAtom-Manipulated Materials Science."

The conference was chaired by R.P.H. Chang, Northwestern University, Takuo Sugano, University of Tokyo, and Van Tran Nguyen, CNET CNS.

Below are highlights from two of the four sessions. For more detailed information on the conference, contact the Materials Research Society for the proceedings.

\section{Optoelectronics}

T. Kobayashi reported on some of the recent progress in the physics of nonlinear optical behavior in organic materials such as polydiacetylene and polythiophene. Such materials are potentially useful for future ultrafast all-optical switching devices. The talk was a refreshing reminder of the long-term potential of materials other than semiconductors, while at the same time showing that much of the basic physics is common to these different fields.

J. Volkl gave an overview of the importance of and issues in substrate materials for optoelectronics, such as GaAs and InP. Some of the issues still relate to the purity of the starting elements. One goal is to make semi-insulating InP without having to dope it with Fe. Recent research has achieved promising results. Uniformity of substrate doping and dislocation density are still very important issues for devices, affecting both yield and performance. Volkl emphasized that achieving significant progress in the difficult task of growing improved substrate materials will require more extensive cooperation in the growth community.

U. Koren discussed the sophisticated growth and fabrication techniques used in making photonic integrated circuits. Such circuits consist of integrated combinations of semiconductor lasers, photodetectors, waveguides, modulators, amplifiers, and filters. This kind of integration will be important for improving the functionality and performance of optoelectronic systems as well as reducing cost.

Koren described one particular process starting with the growth of a complex layer structure including quantum well layers. This single structure can subsequently be processed by a combination of techniques to make several different components together in the same structure. One example circuit contained four different tunable lasers combined through waveguide couplers into an amplifier section to give a wavelength-division-multiplexed optical transmitter.

Other highlights included the remarkable technique reported by E. Yablonovitch of Bellcore in which entire semiconductor films of micron thickness can be floated off their growth substrate and bonded to a variety of other substrates. This may be an important technology for new kinds of hybrid optoelectronic integration.

L.M.F. Chirovsky of AT\&T Bell Laboratories showed $64 \times 32$ arrays of symmetric self-electrooptic effect devices for experimental dimensionally parallel optical switching and processing systems. These devices require a sophisticated layered semiconductor structure that includes both quantum wells and an integral multilayer dielectric mirror grown by molecular beam epitaxy.

J.M. Halbout of IBM discussed siliconbased optoelectronics, including waveguides and detectors for optical receivers. Many demonstrations, including dielectric waveguides, grating couplers, and $\mathrm{Si} / \mathrm{Ge}$ superlattice photodetectors, are promising for future optoelectronic integration with silicon.

Other papers covered novel epitaxial

\section{ICEM II Conference Proceedings}

Hardcover or microfiche,

110 papers, 661 pages.

ISBN: 1-55899-092-5 Code: ICEM-2B

$\$ 55$ MRS Members

$\$ 62$ U.S. List

$\$ 70$ Foreign

Call MRS at (412) 367-3012. growth technologies, techniques for $\mathrm{k}$ doping in very thin sheets for novel electronic and optoelectronic devices, growth of $\mathrm{GaAs}$ light-emitting diodes on silicon substrates, and successful optoelectronic modulator structures using strained layers.

\section{High $\mathrm{T}_{\mathrm{c}}$ Superconducting \\ Electronics}

Wayne Cooke (Los Alamos National Laboratory) presented results on microwave properties of thin films of the cuprate superconductor thin films grown by in-situ laser deposition and sputtering as well as $e x$-situ processed films. The microwave properties and fabrication of microwave devices was also the focus of the talk by Findikoglu et al. They discussed the fabrication, testing, and theory of meanderline microwave resonators. These meanderline resonators were fabricated from laser deposited or sputter YBCO films.

A. Inam (Bellcore) gave an excellent overview of the structure and transport properties of in-situ pulsed-laser-deposited Y-Ba-Cu-O thin films. This invited talk focused primarily on the growth and characterization of epitaxial heterostructures for junction applications. In addition to this, Inam also discussed the microwave properties of YBCO thin films. Other papers addressed other thin film deposition techniques such as MOCVD, plasma enhanced MOCVD, and spray pyrolysis.

J. Geerk (KFK, Karlsruhe, Germany) outlined the dc magnetron deposition technique, including the inverted cylindrical magnetron sputtering pioneered at Karlsruhe. He presented results from a variety of tunneling transport experiments. M. Siegal et al. from AT\&T Bell Laboratories reported the results of systematic variations in the cationic stoichiometry and the growth conditions on the structural and electrical transport properties of ex-situ processed electron beam deposited $\mathrm{YBCO}$ thin films. Asano et al. from NTT (Japan) reported the growth of $\mathrm{YAIO}_{3}$ single crystals which had better microwave properties compared to $\mathrm{LaAlO}_{3}$.

All the oral sessions were followed by a joint poster session. The overall quality of the poster presentations was excellent. There were some interesting posters aimed at the growth of HTSC films on metallic or semiconductor substrates. It was clear, however, that the crystalline quality and electrical properties of such films is still well below that obtained by laser deposition or sputtering onto single crystalline oxide substrates. 


\section{Call for Papers}

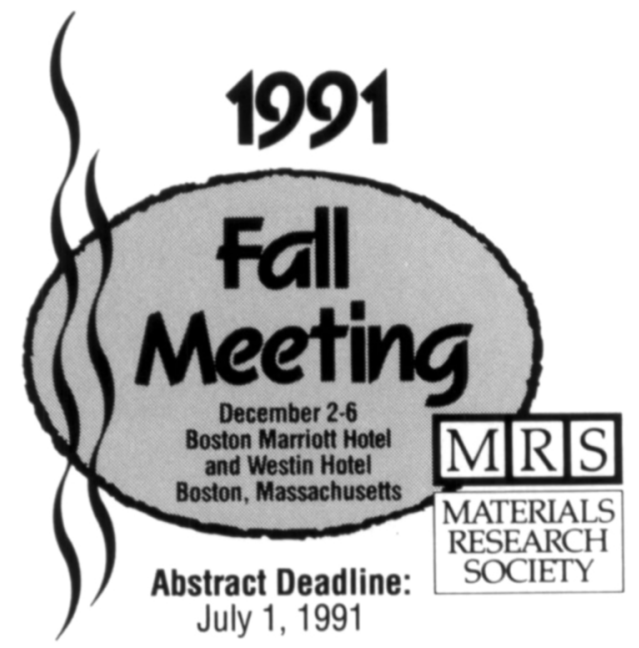

\section{SYMPOSIA}

\section{A: PHASE FORMATION AND MODIFICATION}

\section{BY BEAM-SOLID INTERACTIONS}

Gary S. Was, University of Michigan, (313) 763-4675, FAX (313) 763-4540, Gary S. Was@um.cc.umich.edu; Lynn E. Rehn, Argonne National Laboratory, (708) 972-5021, FAX (708) 972-4798; David M. Follstaedt, Sandia National Laboratories, (505) 844-2102, FAX (505) 844-7775

\section{B: PHOTONS AMD LOW ENERGY PARTICLES IN SURFACE PROCESSING}

Carol Ashby, Sandia National Laboratories, (505) 844-2303, FAX (505) 844-3211; James H. Brannon, IBM Almaden Research Center, (408) 9271626, FAX (408) 927-2100: Stella Pang. University of Michigan, (313) 9362962, FAX (313) 747-1781

\section{Ca: IMTERFACE DYMAMICS AND GROWTH}

Keng S. Liang, Exxon Research and Engineering Company, (908) 7303032, FAX (908) 730-3042; Michael P. Anderson, Exxon Research and Engineering Company, (908) 730-2756, FAX (908) 730-3042; Robijn F. Bruinsma, University of California, Los Angeles, (213) 825-8539, FAX (213) 825-0098; Giacinto Scoles, Princeton University, (609) 258-5570, FAX (609) 258-6746

\section{Ch: STRUCTURE AND PROPERTIES OF INTERFACES IM MATERIALS}

William A.T. Clark, The Ohio State University, (614) 292-2538, FAX (614) 292-1537; Clyde L. Briant, General Electric Corporate Research \& Development, (518) 387-5765, FAX (518) 387-7495; Ulrich Dahmen, Lawrence Berkeley Laboratory, (415) 486-4627, FAX (415) 486-5888
D: THIN FILMS: STRESSES AND MECHANICAL PROPERTIES III

William D. Nix, Stanford University, (415) 725-2605, FAX (415) 725-4034; John C. Bravman, Stanford University, (415) 723-3698, FAX (415) 7254034; Eduard Artt, Max-Planck-Institut für Metallforschung, Germany, (49) 711-2095-219, FAX (49) 711-2095-250; L. Ben Freund, Brown University, (401) 863-1476, FAX (401) 863-1157

\section{E: ADVANCED III-V COMPOUND SEMICONDUCTOR GROWTH, PROCESSING AND DEVICES}

S.J. Pearton, AT\&T Bell Laboratories, (908) 582-4757, FAX (908) 5825917; D.K. Sadana, IBM T.J. Watson Research Center, (914) 945-2423, FAX (914) 945-3688; J.M. Zavada, U.S. Army Research Office, (919) 5490641, FAX (919) 549-4310

\section{F: LOW TEMPERATURE (LT) GaAS AND RELATED MATERIALS}

Gerald L. Witt, AFOSR/NE, (202) 767-4931, FAX (202) 767-0466; Robert Calawa, MIT Lincoln Laboratory, (617) 981-4576, FAX (617) 981-3433; Umesh Mishra, University of California, Santa Barbara, (805) 893-3586, FAX (805) 893-3262; Eicke Weber, University of California, Berkeley, (415) 642-0205, FAX (415) 642-9164

\section{G: WIDE BAND-GAP SEMICONDUCTORS}

Theodore D. Moustakas, Boston University, (617) 353-5431, FAX (617) 353-6440; Jacques I. Pankove, University of Colorado, (303) 492-5470, FAX (303) 492-2758; Y. Hamakawa, Osaka University, Japan, (81) 6-8441151, Ext. 4585, FAX (81) 6-853-1362 


\section{H: HIGH-TEMPERATURE SUPERCONDUCTORS:}

\section{MATERIALS RESEARCH FOR EMERGING TECHNOLOGIES}

Alfredo C. Anderson, MIT Lincoin Laboratory, (617) 981-4708, FAX (617) 981-5328, anderson@il.II.mit.edu; Robert J. Cava, AT\&T Bell Laboratories, (908) 582-2180, FAX (908) 582-2521; Siu Wai Chan, Columbia University, (212) 854-8519, FAX (212) 854-8362; Randy W. Simon, Conductus, Inc., (408) 746-1099, FAX (408) 737-6699; Kiyotaka Wasa, Matsushita Electric Industries Co. Ltd., Japan, (81) 6-906-4860, FAX (81) 6-906-4593

\section{I: FERROELECTRIC THIN FILMS II}

Angus I. Kingon, North Carolina State University, (919) 737-2347, FAX (919) 737-7724; Edward R. Myers, National Semiconductor Corporation, (408) 721-2258, FAX (408) 736-8503; Bruce Tuttle, Sandia National Laboratories, (505) 845-8026, FAX (505) 844-2974

\section{J: OPTICAL WAVEGUIDE MATERIALS}

Matthijs M. Broer, AT\&T Bell Laboratories, (908) 582-7761, FAX (908) 582-2783; H. Kawazoe, Tokyo Institute of Technology, Japan, (81) 45-9221111, Ext. 2357, FAX (81) 45-921-1015; George H.Sigel, Rutgers University, (908) 932-4729, FAX (908) 932-4545; R. Th. Kersten, Schott Glaswerke, Germany, (49) 6131-333-354, FAX (49) 6131-333-341

\section{K: ADVANCED CEMENTITIOUS SYSTEMS: \\ MECHAHISMS ANO PROPERTIES}

F.P. Glasser, University of Aberdeen, United Kingdom, (44) 22-427-2906, FAX (44) 22-427-2921; P.L. Pratt, Imperial College, United Kingdom, (44) 71-589-5111, Ext. 5901, FAX (44) 71-584-7596; T.0. Mason, Northwestern University, (708) 491-3198, FAX (708) 491-7820; J.F. Young, University of Illinois, (217) 244-6210, FAX (217) 244-6917; G.J. McCarthy, North Dakota State University, (701) 237-7193, FAX (701) 237-8831

\section{L: IMNOVATIONS IN THE DEVELOPMENT AND CHARACTERIZATION} OF MATERIALS FOR INFRASTRUCTURE

John M. Barsom, USX Corporation, (412) 433-2147, FAX (412) 433-2148; Jack Youtcheff, NRC/SHRP, (202) 334-1457, FAX (202) 223-2875; Randall P. Bright, Air Products and Chemicals, Inc., (215) 481-3705, FAX (215) 481-4210; Paul Zia, North Carolina State University, (919) 737-7387, FAX (919) $737-7908$

\section{M: SHAPE MEMORY MATERIALS AMD PHEROMENA- \\ FUNDAMENTAL ASPECTS AND APPLICATIONS}

C.T. Liu, Oak Ridge National Laboratory, (615) 574-4459, FAX (615) 5747659; Manfred Wuttig, University of Maryland, (301) 405-5211, FAX (301) 314-9467; K. Otsuka, University of Tsukuba, Japan, FAX (81) 298-55-7440 Henry Kunsmann, Eaton Corporation, (414) 449-7469, FAX (414) 449-6221

\section{N: ELECTRICAL, OPTICAL, AND MAGNETIC PROPERTIES \\ OF ORGANIC SOLID STATE MATERIALS}

Long Y. Chiang, Exxon Research and Engineering Company, (908) 7302656, FAX (908) 730-3042; Anthony F. Garito, University of Pennsylvania, (215) 898-5810, FAX (215) 898-2010; Daniel J. Sandman, GTE Laboratories, Inc., (617) 466-4216, FAX (617) 890-9320

\section{0: COMPLEX FLUIDS}

David Weitz, Exxon Research and Engineering Company, (908) 730-3536 FAX (908) 730-3076; Eric Sirota, Exxon Research and Engineering Company, (908) 730-3407, FAX (908) 730-3076; Tom Witten, University of Chicago, (312) 702-0947, FAX (312) 702-7423; Jacob Israelachvili, University of California, Santa Barbara, (805) 893-8407, FAX (805) 893-4731

P: DISORDERED MATERIALS: FRACTALS, SCALING AND DYMAMICS

Lawrence M. Schwartz, Schlumberger-Doll Research, (203) 431-5210, FAX (203) 438-3819; James V. Maher, University of Pittsburgh, (412) 6249007, FAX (412) 624-9163; Thomas C. Halsey, University of Chicago, (312) 702-7207, FAX (312) 702-5863
Q: SYNTHESIS AND PROCESSING OF CERAMICS: SCIENTIFIC ISSUES Wendell E. Rhine, Massachusetts Institute of Technology, (617) 253-5186, FAX (617) 258-6936; Thomas M. Shaw, IBM T.J. Watson Research Center, (914) 945-3196, FAX (914) 945-4001; R.J. Gottschall, U.S. Department of Energy, (301) 353-3428, FAX (301) 353-6594; Y. Chen, Oak Ridge National Laboratory, (301) 353-3428, FAX (301) 353-6594

\section{R: CHEMICAL VAPOR DEPOSITION OF REFRACTORY} METALS AND CERAMICS

Theodore M. Besmann, Oak Ridge National Laboratory, (615) 574-6852, FAX (615) 574-4913; Bernard M. Gallois, Stevens Institute of Technology, (201) 420-5263, FAX (201) 963-3017; James Warren, Composite Innovation Corporation, (818) 884-0144, FAX (Same as telephone)

S: GAS PRESSURE EFFECTS ON MATERIALS PROCESSIMG AND DESIGN Kozo Ishizaki, Nagaoka University of Technology, Japan, (81) 258-466000 , FAX (81) 258-46-6972; John K. Tien, The University of Texas at Austin, (512) 471-6709, FAX (512) 471-6713; Ed Hodge, Gorham Advanced Materials Institute, (207) 892-5445, FAX (207) 892-2210

\section{T: TISSUE-INDUCING BIOMATERIALS}

Marcy Flanagan, Codman \& Shurtleff, Inc., (617) 986-3215, FAX (617) 986-5285; Linda Cima, Massachusetts Institute of Technology, (617) 2530013, FAX (617) 253-9695; Eyal Ron, Genetics Institute, (617) 876-1170, Ext. 357, FAX (617) 876-1504

\section{U: NEW STRATEGIES FOR THE SYNTHESIS}

\section{AND CHARACTERIZATIOH OF CATALYSTS}

S. Mark Davis, Exxon Research and Development Laboratories, (504) 3597719, FAX (504) 359-8037; Abhaya Datye, University of New Mexic0, (505) 277-0477, FAX (505) 277-0813; Bruce Tatarchuk, Auburn University, (205) 844-2023, FAX (205) 844-2063

\section{V: APPLICATION OF MULTIPLE SCATTERIKG THEORY}

\section{TO MATERIALS SCIENCE}

William H. Butler, Oak Ridge National Laboratory, (615) 574-4845, FAX (615) 574-7659; Peter H. Dederichs, KFA Institut für Festkorperforschung, Germany, (49) 2461-61-4351, FAX (49) 2461-61-2410;A. Gonis, Lawrence Livermore National Laboratory, (415) 422-7150, FAX (415) 422-7300; Richard Weaver, University of Illinois, (217) 333-3656, FAX (217) 244-5707

\section{W: WORKSHOP ON SPECIMEN PREPARATION FOR}

TRANSMISSION ELECTRON MICROSCOPY OF MATERIALS - III

Ron Anderson, IBM East Fishkill Facility, (914) 892-2225, FAX (914) 8922555; John Bravman, Stanford University, (415) 723-3698, FAX (415) 7254034; Bryan Tracy, Intel Corporation, (408) 765-2346, FAX (408) 765-2633

\section{$X$ : FRONTIERS OF MATERIALS RESEARCH}

Rustum Roy, The Pennsylvania State University, (814) 865-3421, FAX (814) $865-2326$

\section{Y: THE ROLE OF NEW MATERIALS AND NEW MATERIAL PROCESSES}

IN THE PROMOTION OF POLUUTION PAEVENTION

Daniel J. Watts, New Jersey Institute of Technology, (201) 596-3465, FAX (201) $802-1946$

\section{2: HIERARCHICAUY STRUCTURED MATERIALS}

Ithan A. Aksay, University of Washington, (206) 543-2625, FAX (206) 5436381, IAKSAY@UWAENG; Eric Baer, Case Western Reserve University, (216) 368-4203, FAX (216) 368-4202, EBAER@CWRUEMAC; Mehmet Sarikaya, University of Washington, (206) 543-0724, FAX (206) 543-6381, MSARIKAY@UWAENG; David A. Tirrell, University of Massachusetts, (413) 545-2680, FAX (413) 545-0082, TIRRELL@UMAECS

\section{MEETING CHAIRS}

Julla M. Phillips, AT\&T Bell Laboratories, Room 1D-158, 600 Mountain Avenue, Murray Hill, NJ 07974-2070; TEL (908) 582-4428, FAX (908) 582-2521

Michael M.J. Treacy NEC Research Institute Inc., 4 independence Way, Princeton, NJ 08540; TEL (609) 951-2613, FAX (609) 951-2496

Man H. You, Oak Ridge National Laboratory, P.O. Box 2008, Oak Ridge, TN 37831-6115; TEL (615) 574-5165, FAX (615) 574-7659 